\title{
Rare Transformation of Chronic Myeloid Leukemia into Acute Lymphoblastic Leukemia in a 14-Year Old Patient
}

\author{
Javera Tariq, Sundas Ali, Shahzad Ali Jiskani*, Rabiah Asghar \\ Department of Pathology, Pakistan Institute of Medical Science (PIMS), Islamabad, Pakistan.
}

\begin{abstract}
Chronic myeloid leukemia is a myeloproliferative neoplasm. It is a common disease of elderly, rarely affecting pediatric, adolescent or young adults. The median age of presentation is 65 years. The incidence is 1-2 cases per 100,000 adults. Tyrosine kinase inhibitors (TKIs) are highly successful and have good remission rates in the CML treatment; though multiple factors are associated with failure of treatment. We present a patient of 14 years old male who was diagnosed as CML. He was seven months into TKI therapy, transformed into B-Lineage acute lymphoblastic leukemia.
\end{abstract}

Keywords: Chronic myeloid leukemia, Rare, Acute lymphoblastic leukemia, Pediatric, Transformation.

\section{INTRODUCTION}

Chronic myeloid leukemia (CML), a myeloproliferative neoplasm, has prevalence rate of 1-2 cases/100,000 adult population. In adult population, out of all newly diagnosed leukemia cases, CML accounts for about $15 \%$. Median age of CML in children patients is about 12.5 years (with range of 1 year to 18 years). Majority of the patients presented have age more than 10 years $[1,2]$.

The median age at the time of diagnosis of CML is about 60 to 65 years and its presentation in children and adolescents is rare. In pediatric age population, CML makes $2 \%$ of all leukemia, and it constitutes approximately $9 \%$ of all leukemic cases in adolescents age population between 15 and 19 years of age, with an yearly incidence of 1 and 2.2 cases/million in these two groups, respectively. There is lack of standardized management guidelines since CML is rare in pediatric population [3].

Chronic myeloid leukemia is explained by a genetic translocation which is balanced and there is binding of Abelson gene (ABL1) (from chromosome 9q34) and the breakpoint cluster region (BCR) gene (from chromosome 22q11.2 $\mathrm{t}(9 ; 22)(\mathrm{q} 34 ; \mathrm{q} 11.2))$. This reorganization is called ''Philadelphia chromosome', Due to this translocation on molecular level, there is the formation of a BCR-ABL1 fusion oncogene that translates into a oncoprotein, BCR-ABL. BCR-ABL is a basically an active tyrosine kinase that is responsible for activation of a number of pathways involved in signal-transduction due to which survival and growth of hematopoietic cells are compromised [4].

\section{CASE REPORT}

A 14 year old boy, resident of Islamabad presented to the OPD with a one week history of low grade fever, bruises all over

*Address correspondence to this author at the Department of Pathology, Pakistan Institute of Medical Science (PIMS), Islamabad, Pakistan.

E-mail: shahzadbaloach289@gmail.com the body and multiple, painful nodules on the legs. There was no significant past medical or surgical history. On examination, he was febrile (temperature of $100^{\circ} \mathrm{F}$ ), pulse rate of $104 / \mathrm{min}$, respiratory rate of $28 / \mathrm{min}$ and a moderate degree of pallor. He had multiple nodules on his legs that were non tender and firm in consistency. Spleen $(5 \mathrm{~cm})$ was palpable. Liver $(4 \mathrm{~cm})$ was palpable, firm in consistency with sharp borders and smooth surface. There was no lymphadenopathy or jaundice. All other systemic examinations were unremarkable.

The complete blood count showed haemoglobin $8.1 \mathrm{gm} / \mathrm{dl}$, Platelet $238,000 / \mu 1$, and TLC $149 \times 10^{9} / 1$. The differential counts showed Neutrophils 56\%, Lymphocytes 08\%, Eosinophils $02 \%$, Monocytes $02 \%$, Myelocytes $19 \%$, Metamyelocytes $12 \%$, bands $02 \%$ and basophils $02 \% .04$ nucleated RBCs were seen per $100 \mathrm{WBCs}$ (Fig. 1).

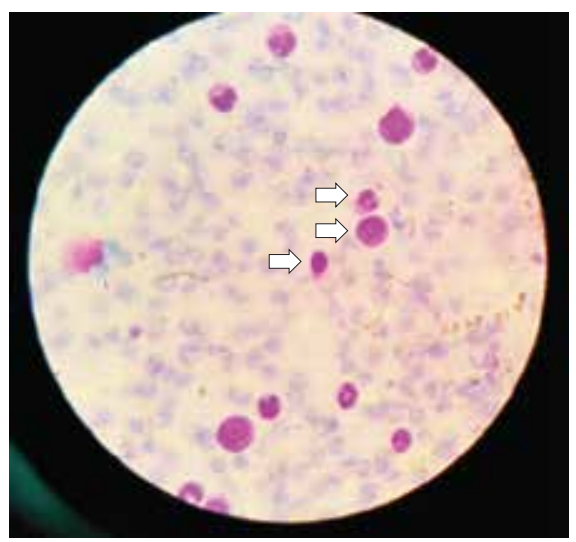

Fig. (1). Peripheral Film: Bimodal Peak of Mature Neutrophils and Myelocytesa Long with a Basophil (Wright x 1000).

Peripheral smear showed anisocytosis, poikilocytosis, and leukocytosis showing bimodal peak of mature neutrophils and myelocytes. Reticulocytes were $0.3 \%$. Ultrasound Abdomen showed hepatosplenomegaly. Bone marrow examination

www.njhsciences.com 
showed a very hypercellular smear (Fig. 2). Myeloid series was very hypercellular with bimodal peak (Fig. 3). Erythropoiesis was relatively depressed with normoblastic maturation. M: E ratio was 15:1.Dwarf forms of megakaryocytes were seen (Fig. 4).

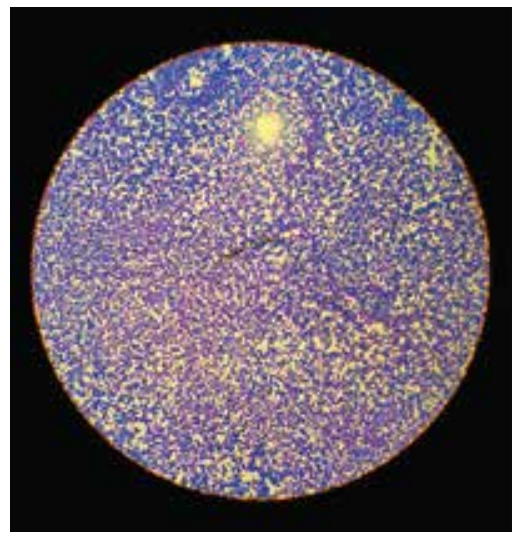

Fig. (2). Bone Marrow Aspirate Showing Marked Hypercellularityon Low Power (Wright x 100).

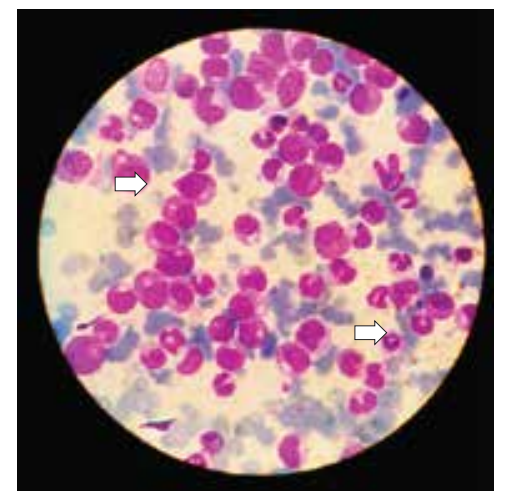

Fig. (3). Bone Marrow Aspirate: Bimodal peak of Myelocytes and Mature Neutrophils (Wright x 1000).

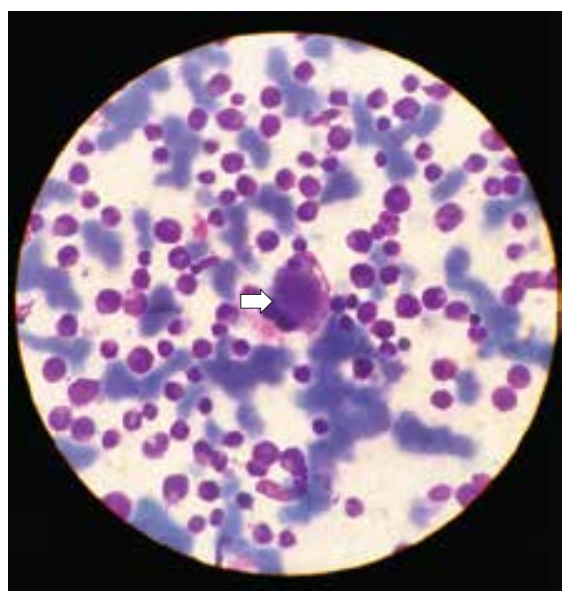

Fig. (4). Bone Marrow Aspirate: Dwarf Megakaryocyte (Wright x 1000).
Based on these findings, a final diagnosis of Myeloproliferative Neoplasm, most likely chronic myeloid leukemia (chronic phase) was made. On quantitative PCR, BCR-ABL1 mRNA was identified with a detection limit of $71 \%$ (IS). Treatment was started with Imatinib400 mg PO QID.

Seven months into the therapy patient suddenly developed extreme malaise, fever and diarrhea. When blood CP was performed it showed Hemoglobin $8.4 \mathrm{gm} / \mathrm{dl}$, Platelet count $13000 / \mu \mathrm{l}$ and TLC $267 \times 10^{9} / \mathrm{L}$. Peripheral smear showed normocytic normochromic anemia, thrombocytopenia with leukocytosis and $88 \%$ blasts. Flow cytometry on peripheral blood was performed in which the gated population was positive for weak CD45(94\%), CD19+34(66\%), CD10+19(90\%), CD13(0\%), CD33(0\%), HLA DR (2\%), CD7(4\%), CD3(3\%), CD5(3\%), CD14(0\%) consistent with $\mathrm{B}$ cell lineage Acute lymphoblastic leukemia.

\section{DISCUSSION}

Chronic myeloid leukemia is a Myeloproliferative Neoplasm (MPN) in which the prominent proliferative component is the granulocytes. Untreated CML has either bi or triphasic natural history; an early chronic phase that precedes an accelerated or blast phase, or both. CML is very uncommon in children and very small population (5\%) presents at advanced stage. The risk factors for $\mathrm{CML}$ are unknown. However, exposure to radiation is thought to play a role. There is very little hereditary predisposition for development of CML. Common presenting symptoms include malaise, fatigue, weight loss, night sweats, and anemia, and splenomegaly is present in almost 50\% [5].

Patients presenting with chronic myeloid leukemia below the age of 20 years present with hyperleukocytosis. In children, total white blood cell count is approximately twice at the time of diagnosis than adults, the division of blasts, myelocytes and promyelocytes, is considerably higher, and hyperleukocytosis and its clinical manifestations are far more common in pediatric age group as compared to adults [6]. The detection of $\mathrm{Ph}$ chromosome and/or BCR-ABL 1 is required for diagnosis of CML. The progression of CML in chronic phase to blast crises either directly or through accelerated phase occurs in around 3-5 years post diagnosis [5].

In international registry of childhood chronic myeloid leukemia (7.5\%) children presented at initial diagnosis with $\mathrm{CML}$ in advance phase in accordance with European LeukemiaNet criteria out of whom (4\%) patients were diagnosed in accelerated phase and 3.5\% diagnosed in blastic phase in which $70 \%$ had lymphoid immunophenotype [7].

Hossain et al. reported two cases showing typical features of CML except for the age group that belonged to much unusual young adult group for the first case and pediatric age group for the second case [8]. 
Many pediatric oncologists treat the pediatric CML patients on treatment guidelines devised for adult patients and in many countries like Pakistan adult oncologists treat children suffering from CML [3].

\section{CONCLUSION}

Since there are very few cases of CML in pediatric population and adult oncologists are treating children on the same guidelines, there is a need of training pediatric oncologist to treat CML. In addition special treatment guidelines must be devised keeping in mind the variance of disease in children.

\section{CONFLICT OF INTEREST}

Declared none.

\section{ACKNOWLEDGEMENTS}

We acknowledge all our fellow colleagues.

\section{AUTHORS' CONTRIBUTION}

Javera Tariq: Concept, Manuscript Writing, Discussion.

Sundas Ali: Experimentation, Study Designing, Discussion.

Shahzad Ali Jiskani: Interpretation, Discussion, Planning.

Rabiah Asghar: Analysis, Discussion.

\section{REFERENCES}

[1] Huho AN, Issaq N, Iacobas I, et al. A Rare case of pediatric chronic myelogenous leukemia presenting with severe throm- bocytosis without leukocytosis. Pediatr Dev Pathol 2018; 21(1): 100-4. DOI: $10.1177 / 1093526617698601$

[2] Jabbour E, Kantarjian H. Chronic myeloid leukemia: 2018 update on diagnosis, therapy and monitoring. Am J Hematol 2018; 93(3): 442-59. DOI: 10.1002/ajh.25011

[3] Hijiya N, Schultz KR, Metzler M, Millot F, Suttorp M. Pediatric chronic myeloid leukemia is a unique disease that requires a different approach. Blood 2016; 127(4): 392-9.

DOI: $10.1182 /$ blood-2015-06-648667

[4] Bonifacio M, Stagno F, Scaffidi L, Krampera M, Di Raimondo F. Management of chronic myeloid leukemia in advanced phase. Front Oncol 2019; 9: 1-18.

DOI: $10.3389 /$ fonc. 2019.01132

[5] Swerdlow SH, Campo E, Harris NL, Jaffe ES, Pileri SA, Stein H, Eds. WHO Classification Of Tumors Of Hematopoietic And Lymphoid Tissues. Lyon, France: IARC Publication 2017.

[6] Kaushansky K, Lichtman MA, Prchal JT, et al, Eds. Williams Hematology. 9th edn. USA: McGraw-Hill education 2016.

[7] Millot F, Maledon N, Guilhot J, Güneş AM, Kalwak K, Suttorp M. Favourable outcome of de novo advanced phases of childhood chronic myeloid leukaemia. Eur J Cancer 2019; 115: 17-23. DOI: 10.1016/j.ejca.2019.03.020

[8] Hossain MZ, Hassan P, Ahmed B, et al. Two cases of chronic myeloid leukemia in pediatric and young adolescent age group. J Dhaka Med Coll 2017; 25(1): 74-6.

DOI: 10.3329/jdmc.v25i1.33961. 